\title{
Predictors of treatment failure during the first year in newly diagnosed type 2 diabetes patients: an observational study
}

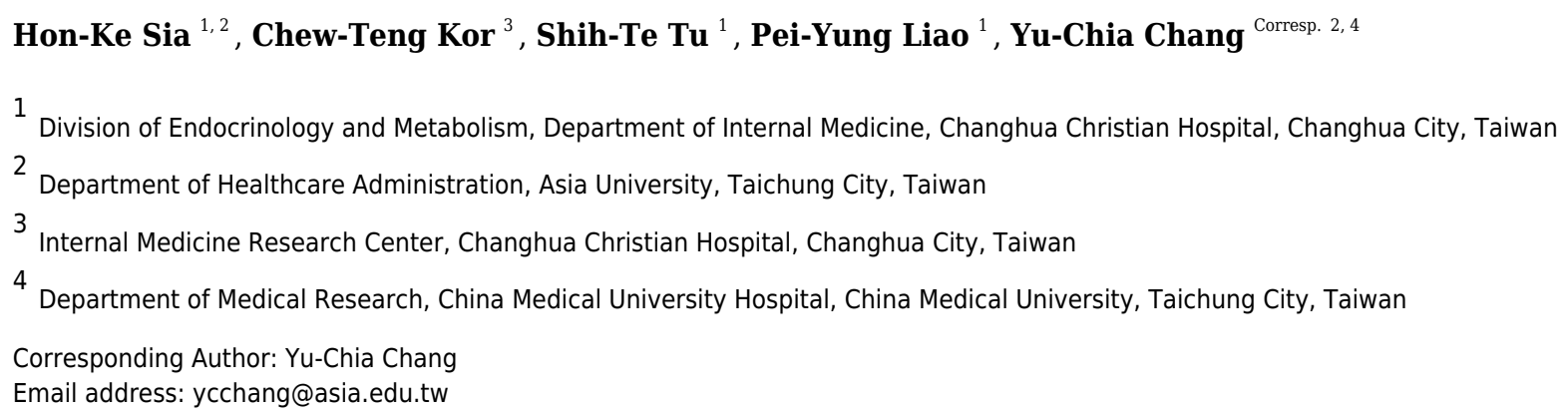

Background. Diabetic patients who fail to achieve early glycemic control may increase the future risk of complications and mortality. The aim of the study was to identify factors that predict treatment failure (TF) during the first year in adults with newly diagnosed type 2 diabetes mellitus (T2DM). Methods. This retrospective cohort study conducted at a medical center in Taiwan enrolled 4282 eligible patients with newly diagnosed T2DM between 2002 and 2017. Data were collected from electronic medical records. TF was defined as the $\mathrm{HbAlc}$ value $>7 \%$ at the end of 1-year observation. A subgroup analysis of 2392 patients with baseline $\mathrm{HbAlc} \geq 8 \%$ was performed. Multivariable logistic regression analysis using backward elimination was applied to establish prediction models. Results. Of all study participants, 1439 (33.6\%) were classified as TF during the first year. For every $1 \%$ increase in baseline $\mathrm{HbAlc}$, the risk of TF was 1.17 (95\% Cl 1.15-1.20) times higher. Patients with baseline $\mathrm{HbAlc} \geq 8 \%$ had a higher rate of TF than those with $\mathrm{HbAlc}<8 \%$ (42.0 vs $23.0 \%, p<0.001$ ). Medication adherence, self-monitoring of blood glucose (SMBG), regular exercise, gender (men), non-insulin treatment, and enrollment during 2010-2017 predicted a significant lower risk of TF in both of the primary and subgroup models.

Conclusions. Newly diagnosed diabetes patients with baseline $\mathrm{HbAlc} \geq 8 \%$ did have a much higher rate of TF during the first year. Subgroup analysis for them highlights the important predictors of TF, including medication adherence, performing SMBG, regular exercise, and gender, in achieving glycemic control. 
1 Predictors of treatment failure during the first year in newly diagnosed type 2 diabetes

2 patients: an observational study

3

4 Hon-Ke Sia1,2, Chew-Teng Kor ${ }^{3}$, Shih-Te Tu1, Pei-Yung Liao1, Yu-Chia Chang2,4

$6{ }^{1}$ Division of Endocrinology and Metabolism, Department of Internal Medicine, Changhua

7 Christian Hospital, Changhua City, Taiwan

$8{ }^{2}$ Department of Healthcare Administration, Asia University, Taichung City, Taiwan

9 3nternal Medicine Research Center, Changhua Christian Hospital, Changhua City, Taiwan

$10{ }^{4}$ Department of Medical Research, China Medical University Hospital, China Medical University,

11 Taichung City, Taiwan

12

13 Corresponding Author:

14 Yu-Chia Chang

15500 Lioufong Rd., Wufeng, Taichung City 41354, Taiwan

16 Email address: ycchang@asia.edu.tw

18 Abstract

19 Background. Diabetic patients who fail to achieve early glycemic control may increase the

20 future risk of complications and mortality. The aim of the study was to identify factors that

21 predict treatment failure (TF) during the first year in adults with newly diagnosed type 2 diabetes

22 mellitus (T2DM).

23 Methods. This retrospective cohort study conducted at a medical center in Taiwan enrolled 4282 
24 eligible patients with newly diagnosed T2DM between 2002 and 2017. Data were collected from 25 electronic medical records. TF was defined as the HbAlc value $>7 \%$ at the end of 1 -year 26 observation. A subgroup analysis of 2392 patients with baseline $\mathrm{HbA} 1 \mathrm{c} \geq 8 \%$ was performed.

27 Multivariable logistic regression analysis using backward elimination was applied to establish 28 prediction models.

29 Results. Of all study participants, 1439 (33.6\%) were classified as TF during the first year. For 30 every $1 \%$ increase in baseline $\mathrm{HbAlc}$, the risk of TF was 1.17 (95\% CI 1.15-1.20) times higher.

31 Patients with baseline $\mathrm{HbA} 1 \mathrm{c} \geq 8 \%$ had a higher rate of TF than those with $\mathrm{HbA} 1 \mathrm{c}<8 \%(42.0$ vs 32 23.0\%, $<<0.001$ ). Medication adherence, self-monitoring of blood glucose (SMBG), regular 33 exercise, gender (men), non-insulin treatment, and enrollment during 2010-2017 predicted a 34 significant lower risk of TF in both of the primary and subgroup models.

\section{Conclusions.}

36 Newly diagnosed diabetes patients with baseline $\mathrm{HbA} 1 \mathrm{c} \geq 8 \%$ did have a much higher rate of $\mathrm{TF}$ 37 during the first year. Subgroup analysis for them highlights the important predictors of TF, 38 including medication adherence, performing SMBG, regular exercise, and gender, in achieving 39 glycemic control.

\section{Introduction}

42 Diabetes mellitus (DM) is among the most serious chronic diseases worldwide. The 43 prevention and treatment of diabetes is a major health care issue due to its high prevalence, 44 related comorbidities, complications, and high related medical cost. Early glycemic control may 45 have long-lasting (at least 10 years) effects in reducing the risk of severe microvascular and 46 macrovascular complications, known as the legacy effect (metabolic memory) [1, 2]. Walraven 
47 et al. reported that patients who responded quickly to glycemic control showed a lower

48 prevalence of retinopathy and microalbuminuria [3]. A large cohort study of newly diagnosed diabetes patients with at least 10 -year survival showed that poor control (mean $\mathrm{HbA1c} \geq 8.0 \%$ )

50

51

52

53

54

55

56

57

64 65

68 during the first year was associated with increased future risk of microvascular events and mortality [4]. These findings highlight the urgency of improving glycemic control in newly diagnosed diabetes patients.

Despite a tendency for better islet function in newly diagnosed patients with type $2 \mathrm{DM}$ (T2DM), many still fail to achieve early glycemic control. A nationwide prospective cohort study reported that $31.5 \%$ of newly diagnosed Chinese diabetes patients failed to achieve HbA1c target levels $(<7.0 \%)$ after 12 months of treatment [5]. Early detection of the factors that predispose to treatment failure could help identify those at risk of not achieving glycemic control and enable tailoring of treatment measures.

Previous studies investigating predictors of poor glycemic control rarely focused on newly diagnosed T2DM patients $[5,6]$. There exist characteristic differences between newly diagnosed patients and those who had been on long-term treatment; thus, their predictors may also differ. Ren et al. reported that predictors of the response to anti-diabetic therapy differed between earlyand advanced-stage T2DM [7]. The findings of interventional studies may not reflect the situation in clinical practice, particularly medication adherence $[8,9]$. Therefore, further studies focusing on newly diagnosed patients using real-world data are required to fill this information gap. The aim of the present study was to determine the major factors predicting treatment failure during the first year in adults with newly diagnosed T2DM.

\section{Materials \& Methods}




\section{Subjects}

71 This retrospective cohort study was conducted at the Changhua Christian Hospital (CCH),

72 Taiwan. A total of 24473 patients with T2DM were enrolled in the diabetes case management

73 program (DCMP) at the CCH Diabetes Care Centre between January 2002 and December 2017.

74 Patients were screened for eligibility using data from the hospital's electronic medical record 75 system.

76 Patients diagnosed with T2DM, according to the criteria established by the American Diabetes 77 Association, were included [10]. Those in whom the onset of diabetes occurred over 12 months 78 prior to enrolment or at an age $<30$ years were excluded. The latter was to reduce the likelihood 79 of type 1 diabetes. Patients aged $\geq 65$ years or with a Charlson comorbidity index scores $\geq 5$ were 80 excluded $[11,12]$, considering that less stringent $\mathrm{HbA} 1 \mathrm{C}$ goals (such as $8-8.5 \%$ ) have been 81 recommended for patients with limited life expectancy, extensive comorbid conditions, or frail, 82 older adults since late 2000s [13, 14]. Patients with estimated glomerular filtration rate (eGFR) $83<30 \mathrm{~mL} / \mathrm{min} / 1.73 \mathrm{~m}^{2}$ were also excluded as this may have affected the HbA1c level and not 84 accurately reflect the true glycemic status [15]. In the end, 4282 eligible patients with $>1$ year of 85 analytical data were included (Figure 1).

\section{Data collection}

88 Data collected from the hospital's electronic medical record system included the DCMP 89 diabetes registry, prescriptions, laboratory data, and $\mathrm{CCH}$ research database. Diabetes specialists 90 referred patients with T2DM to the Diabetes Care Center to participate in the DCMP, usually 2

91 to 6 weeks after the first outpatient clinic visit. All patients received basic data registry,

92 underwent health-related behavior survey, physical examination, and laboratory testing. They 
93 attended standardized one-to-one diabetes self-management (DSM) education classes upon

94 enrolment into the DCMP. After completing the course, a certified diabetes educator conducted

95 face-to-face interviews and evaluated and recorded each patient's frequency of performing Self-

96 monitoring of blood glucose (SMBG), knowledge regarding glycemic control, willingness

97 toward DSM, and medication adherence.

98

99 Outcome measurement

100 Treatment failure (TF) was defined as the $\mathrm{HbAlc}$ value $>7 \%$ at the end of 1 -year observation.

101 The others with $\mathrm{HbA} 1 \mathrm{c}$ levels $\leq 7 \%$ at the end point were categorized as non-TF (reference

102 group). Serum HbA1c was measured through ion-exchange high-performance liquid

103 chromatography using the VARIANTTM II Turbo system.

104

105 Other Variables

106 Basic data included age at onset of diabetes, gender, level of education, and family history of

107 diabetes. Health-related behaviors included current smoking (tobacco use within the preceding

108 year), drinking (alcohol consumption more than once per week within the preceding year), and

109 physical activity [regular ( $\geq 30 \mathrm{~min} /$ day, $\geq 3$ days/week), occasional (low level of exercise less

110 than the regular exercise criteria) or no exercise]. SMBG was defined as self-assessment of blood

111 glucose levels using a glucometer more than once per week. Knowledge regarding glycemic

112 control was defined as an understanding of the need for and methods of controlling blood

113 glucose. Willingness toward DSM was defined as the motivation to learn self-management

114 techniques. Medication adherence was defined as taking medication regularly at the dose

115 recommended by the physician over the past week. Four-point scales were used to assess the 
116 three aforementioned variables. Data were merged into simple dichotomies (i.e., top-two-box vs.

117 bottom-two-box) and categorized as adequate (yes) or inadequate (no) for analysis.

118 Physical examination included measurement of blood pressure (BP), height, and body weight.

119 Systolic BP and diastolic BP were measured with patients in a seated position after a 10-min rest.

120 The mean BP was calculated as (1/3 SBP + 2/3 DBP). Body mass index (BMI) was calculated as

121 body weight $(\mathrm{kg}) /$ height $\left(\mathrm{m}^{2}\right)$. Baseline laboratory data, including total cholesterol (TC), high-

122 density lipoprotein cholesterol (HDL-C), triglycerides (TG), low-density lipoprotein cholesterol

123 (LDL-C), creatinine, and glutamic pyruvic transaminase (GPT) levels were measured using a

124 UniCel DxC 800 Synchron Clinical System (Beckman Coulter, Brea, CA, USA). The eGFR was

125 calculated using the equation recommended by the National Kidney Foundation [16].

126 Individual anti-diabetic medication use during the first six months was categorized as oral 127 anti-diabetic drugs (OAD) alone, insulin alone, both, or none. Only medication used for $>1$

128 month was included. Data on the 19 major non-psychiatric comorbidities in the Charlson

129 comorbidity index during the year preceding enrolment were collected for each patient from the

$130 \mathrm{CCH}$ research database. Major comorbidities including congestive heart failure, coronary artery

131 disease, and cerebrovascular accident were analyzed as independent variables. Enrollment time

132 was classified into two categories: 2002-2009 and 2010-2017.

134 Statistical analysis

135 Data were expressed as frequency with percentage and mean \pm standard deviation for 136 categorical and continuous covariates respectively. Univariable logistic regression analysis was 137 performed to calculate odds ratios (ORs) of TF vs non-TF for all variables. Subsequently, 138 multivariable logistic regression analysis was performed to establish prediction models adjusted 
139 for significant covariates as shown in Table 1. The backward stepwise regression was performed

140 to be a variable selection method to avoid overfitting. We used tolerance and variance inflation

141 factor (VIF) to detect whether there is multicollinearity between covariates in every model. If

142 the value of VIF is less than 2, the multicollinearity problem is considered absent. Area under

143 the receiver operating characteristic curve (AUC) and R-square were used to assess the

144 predictive ability of the models for predicting TF. We performed a subgroup analysis of patients

145 with baseline $\mathrm{HbA} 1 \mathrm{c} \geq 8 \%$ to demonstrate the effect of initial poor glycemic status on TF. All

146 tests were two-tailed with a significance level of 0.05. IBM SPSS Statistics version 22 (IBM

147 Corp., Armonk, NY, USA) was used for the analyses.

148

149 Ethics statement

150 The study was approved by the Institutional Review Board of Changhua Christian Hospital

151 (CCH IRB No: 191212). Informed consent was waived.

152

153 Results

154 We identified 4282 eligible patients (mean age, $50.9 \pm 8.5$ years; $55.1 \%$ males) between 2002 155 and 2017. Among these patients, 1439 (33.6\%) were categorized as the TF group. Compared 156 with the non-TF group, the TF group was younger (50.2 vs 51.3 years, $p<0.001)$ and included 157 more current smokers $(21.5 \%$ vs $17.3 \%, p<0.001)$, whereas the distribution of BMI, alcohol 158 drinking, eGFR, GPT, use of statins, and comorbidities were similar. Patients had lower levels of 159 education, had no family history of diabetes, and women were predisposed to TF (Table 1).

160 Higher baseline HbA1c level, lipid levels (TC, HDL-C, LDL-C and TG), and mean BP 161 indicated higher risk of TF. For every $1 \%$ the increase in baseline HbA1c, the risk of TF was 
1621.17 (95\% CI 1.15-1.20) times higher. Use of fibrates and insulin (alone or combined with OAD)

163 during the first 6 months predicted greater TF. Enrollment during 2010-2017, regular exercise,

164 good medication adherence, performing SMBG, good knowledge regarding glycemic control,

165 and adequate willingness toward DSM reduced risk of TF.

166 According to baseline HbA1c level, the study subjects were divided into two subgroups. The

167 higher HbA1c subgroup was composed of 2392 patients with $\mathrm{HbA1c} \geq 8 \%$, including 1005

168 (42.0\%) with TF. In contrast, only $434(23.0 \%)$ of the 1890 patients with $\mathrm{HbA} 1 \mathrm{c}<8 \%$ had TF

169 during the first year. Therefore, two prediction models were established: the primary model,

170 which consisted of all study subjects, and the subgroup model, which consisted of a subgroup of

171 patients with baseline $\mathrm{HbA} 1 \mathrm{c} \geq 8.0 \%$, using multivariable backward stepwise logistic regression

172 analysis (Table 2). Collinearity diagnostic tests showed an absence of multicollinearity between

173 factors, where values for tolerance ranged from 0.597 to 0.993 , corresponding to VIFs of 1.675

174 to 1.007 (Supplementary Table). Men, regular exercise, performing SMBG, medication

175 adherence and enrollment during 2010-2017 predicted a lower risk of TF in both models. Higher

176 baseline $\mathrm{HbA1c}$, younger age at onset, lower levels of education, and higher mean BP increased

177 the risk of TF in the primary model, but the increase was not statistically significant in the

178 subgroup model. Using insulin within the first 6 months was predictive of TF. Although high TG

179 indicated a higher risk of TF in the primary model, it was replaced by high TC in the subgroup

180 model.

181

182 Discussion

183 Previous studies on predictive factors or model of newly diagnosed T2DM were

184 predominantly based on baseline $\mathrm{HbA} 1 \mathrm{c}$, which is a strong major predictor $[3,5,6,17,18]$. 
185 Higher baseline HbAlc may reflect poor beta cell function or prolonged hyperglycemia due to 186 delayed diagnosis of DM $[6,17]$. Consistent with aforementioned studies, patients with baseline $187 \mathrm{HbAlc} \geq 8 \%$ had a higher rate of TF than those with $\mathrm{HbAlc}<8 \%$ (42.0 vs $23.0 \%, \mathrm{p}<0.001)$. 188 However, it is worth noting that baseline $\mathrm{HbAlc}$ became an insignificant predictor in the 189 subgroup model after adjusting for other factors. In other words, further increase in baseline $190 \mathrm{HbA} 1 \mathrm{c} \geq 8 \%$ may raise a limited risk of TF. Other factors, including gender, SMBG, medication 191 adherence, and regular exercise may be more predictive in newly diagnosed patients with 192 baseline $\mathrm{HbAlc} \geq 8 \%$.

193 Medication non-adherence is common and may account for up to $75 \%$ of the gap in clinical 194 efficacy between randomized controlled trial and real-world results in HbAlc reduction [19, 20]. 195 The present study showed that medication adherence is associated with a greater protection than 196 other modifiable variables, especially in the subgroup model, indicating it may be more 197 influential in reducing the risk of TF in patients with baseline $\mathrm{HbAlc} \geq 8 \%$. It supports clinicians 198 to aggressively promote patients' medication adherence, especially those with high baseline 199 HbA1c.

200 SMBG has been shown to improve glycemic control among diabetes patients using insulin, 201 although its value for those with non-insulin-treated T2DM has remained inclusive [21]. Our 202 study demonstrated that performing SMBG is associated with lower risk of TF in patients with 203 newly diagnosed T2DM, which supports the international Diabetes Federation guideline 204 recommending SMBG should be considered at the time of diagnosis for patients with T2DM as a 205 part of their education [22].

206 Higher level of education was positively correlated with good medication adherence, SMBG, 207 adequate knowledge regarding glycemic control, willingness toward DSM, and regular exercise 
208 (Table 3). Our findings are consistent with those of a previous study in Taiwan that showed that 209 higher educational attainment was significantly associated with better understanding of health 210 education and instructions, adequate health literacy, and better glycemic control [23]. Knowledge 211 regarding glycemic control and willingness toward DSM were not significant predictors in both 212 of the primary and subgroup analyses, indicating that self-care behaviors (such as medication 213 adherence, performing SMBG, and regular exercise) are more predictive of TF than knowledge 214 or willingness in our models.

215 Smoking has been shown to increase diabetes incidence in general population [24]. Additional, 216 an adverse association between smoking and glycemic control has been reported [25]. Smoking 217 may affect glucose homeostasis through several mechanisms, including increased insulin 218 resistance, reduced insulin action, and loss of islet $\beta$-cell mass [25, 26]. Our finding highlights 219 that smoking is associated with increased possibility of TF in patients with newly diagnosed 220 T2DM. Therefore, taking action to the modifiable unhealthy behavior is essential for this 221 population.

222 Our study showed that women are more likely to have TF. The finding is in line with a 223 longitudinal study of 1450 Chinese with diabetes in Hong Kong that reported patients with 224 unimproved control had a female preponderance [27]. Another Germany multicenter study of 2259108 patients with T2DM showed that men had greater HbA1c reduction than women after 226 treatment [28]. By contrast, other studies have shown that women more likely to have glycemic 227 control in comparison with men [29, 30]. Gender differences in glycemic control may involve 228 biological, psychosocial factors, health behaviors, responses to therapeutic interventions, self229 diabetes management, and differential perception and impact of social support [28, 31]. We 230 suggest that gender difference should be considered in treating patients with newly diagnosed 
231 diabetes.

232 The present study showed that older age reduced the risk of TF in newly diagnosed T2DM 233 patients, which was consistent with most previous studies [3, 17, 19, 32]. While older patients 234 tended to have more unfavourable factors, such as less knowledge regarding glycemic control, 235 less likely to perform SMBG and lower level of education, they had a lower risk of TF in the first 236 year (Table 3). There remains a lack of consensus on the mechanisms underlying the inverse 237 association of age and HbA1c. It may involve age-related differences in the pathogenesis of 238 T2DM [32, 33, 34, 35]. Previous studies showed insulin therapy, either alone or combined with $239 \mathrm{OAD}$, was associated with a higher risk of $\mathrm{TF}[5,36]$. A common explanation is that insulin 240 users have more severe beta cell loss and are therefore prone to TF.

241 The strengths of this study include its large sample size, the focus on newly diagnosed 242 T2DM and further identification of predictors in patients with baseline $\mathrm{HbA} 1 \mathrm{c} \geq 8 \%$. The 243 National Health Insurance in Taiwan covers more than 99\% of the country's 23 million people 244 and provides easy access to medical services [37]. Therefore, the treatment and outcome in the 245 study were less affected by insurance factors.

246 Our study had several limitations. First, the study participants were enrolled across a long-

247 time window, and thus available first-line pharmacological treatment options varied considerably.

248 We incorporated enrollment time as a variable in the regression models to reduce the

249 confounding effect. Second, patients attending a medical centre may have higher disease severity.

250 Therefore, we adjusted relevant variables for comorbidity and performed subgroup analysis of

251 patients with $\mathrm{HbA} 1 \mathrm{c} \geq 8 \%$ to reduce the selection bias. Third, the occurrence of TF during the

252 first-year may reflect either a specific medication intolerance, that often prevents patients

253 maintaining their therapy, or therapeutic inertia, which is a non-negligible problem among 
254 diabetes care providers. Additionally, income, dietary habits, and occupation may also contribute

255 to glycemic control. Our models were limited by the absence of related information.

256 Fourth, selection bias may exist since our study population did not include those patients with

257 missing data or $<1$-year follow-up. Fifth, some of the data were self-reported, such as medication

258 adherence and SMBG frequency, and social desirability bias could be a problem. Finally, the

259 generalizability of the real-world study findings may be limited to settings with similar medical

260 and sociocultural environment.

261

262

Conclusions

263 Baseline $\mathrm{HbA1c}$ has been an important indicator in clinical treatment guidelines to assess the 264 severity of glycemic control and guide clinicians to use initial OAD combination therapy or even 265 insulin therapy [38]. The current study showed that patients with baseline $\mathrm{HbA1c} \geq 8 \%$ did have 266 a much higher rate of TF. However, subgroup analysis for them demonstrated that when baseline $267 \mathrm{HbA} 1 \mathrm{c}$ above $8 \%$, the increase in HbA1c did not further raise the risk of TF. Other factors, 268 including medication adherence, regular exercise, performing SMBG, using insulin, and gender 269 became more predictive than baseline HbA1c level.

270

\section{References}

272 [1] Chalmers J, Cooper ME. UKPDS and the legacy effect. N Engl J Med 2008;359:1618-20. 273 doi: 10.1056/NEJMe0807625

274 [2] Holman RR, Paul SK, Bethel MA, et al. 10-year follow-up of intensive glucose control in 275 type 2 diabetes. $N$ Engl J Med 2008;359:1577-89. doi: 10.1056/NEJMoa0806470

276 [3] Walraven I, Mast MR, Hoekstra T, et al. Distinct HbA1c trajectories in a type 2 diabetes 277 cohort. Acta Diabetol 2015;52:267-75. doi: 10.1007/s00592-014-0633-8

278 [4] Laiteerapong N, Ham SA, Gao Y, et al. The legacy effect in type 2 diabetes: impact of early 
279 glycemic control on future complications (the Diabetes \& Aging Study). Diabetes Care 280 2019;42:416-26. doi: 10.2337/dc17-1144

281 [5] Cai X, Hu D, Pan C, et al. The risk factors of glycemic control, blood pressure control, lipid 282 control in Chinese patients with newly-diagnosed type 2 diabetes: A nationwide prospective 283 cohort study. Sci Rep 2019;9:7709. doi:10.1038/s41598-019-44169-4

284 [6] Svensson E, Baggesen LM, Thomsen RW, et al. Patient-level predictors of achieving early 285 glycaemic control in Type 2 diabetes mellitus: a population-based study. Diabet Med 286 2016;33:1516-23. doi: 10.1111/dme.13184

287 [7] Ren Q, Ji LN, Lu JM, et al. Search for clinical predictors of good glycemic control in patients 288 starting or intensifying oral hypoglycemic pharmacological therapy: A multicenter prospective 289 cohort study. J Diabet Complicat 2020;34:107464. doi:10.1016/j.jdiacomp.2019.107464

290 [8] Blonde L, Khunti K, Harris SB, et al. Interpretation and impact of real-world clinical data for 291 the practicing clinician. Adv Ther 2018;35:1763-74. doi: 10.1007/s12325-018-0805-y

292 [9] Edelman SV, Polonsky WH. Type 2 diabetes in the real world: the elusive nature of glycemic 293 control. Diabetes Care 2017;40:1425-32. doi: 10.2337/dc16-1974

294 [10] American Diabetes Association. Classification and diagnosis of diabetes: standards of 295 medical care in diabetes-2019. Diabetes Care 2019;42(Suppl 1):S13-28. doi: 10.2337/dc19296 S002

297 [11] Charlson ME, Pompei P, Ales KL, et al. A new method of classifying prognostic 298 299 comorbidity in longitudinal studies: development and validation. J Chronic Dis 1987;40:373-83. doi: 10.1016/0021-9681(87)90171-8

300 [12] Bannay A, Chaignot C, Blotière PO, et al. The best use of the Charlson Comorbidity Index 301 with electronic health care database to predict mortality. Med Care 2016;54(2):188-194.

302 doi:10.1097/MLR.0000000000000471

303 [13] American Diabetes Association. 6. Glycemic targets: standards of medical care in diabetes304 2021. Diabetes Care 2021;44(Suppl 1):S73-S84. doi:10.2337/dc21-S006

305 [14] American Diabetes Association. 12. Older adults: standards of medical care in diabetes306 2021. Diabetes Care 2021;44(Suppl 1):S168-S179. doi:10.2337/dc21-S012

307 [15] Bloomgarden Z, Handelsman Y. How does CKD affect HbA1c? J Diabetes 2018;10:270. 308 doi: $10.1111 / 1753-0407.12624$

309 [16] Levey AS, Coresh J, Balk E, et al. National Kidney Foundation. National Kidney 
310 Foundation practice guidelines for chronic kidney disease: evaluation, classification, and

311 stratification. Ann Intern Med 2003;139:137-47. doi: 10.7326/0003-4819-139-2-200307150-

31200013

313 [17] Laiteerapong N, Karter AJ, Moffet HH, et al. Ten-year hemoglobin A1c trajectories and 314 outcomes in type 2 diabetes mellitus: the Diabetes \& Aging Study. J Diabet Complicat

315 2017;31:94-100. doi: 10.1016/j.jdiacomp.2016.07.023

316 [18] Hertroijs DFL, Elissen AMJ, Brouwers M, et al. A risk score including body mass index, 317 glycated haemoglobin and triglycerides predicts future glycaemic control in people with type 2 318 diabetes. Diabetes Obes Metab 2018;20:681-88. doi: 10.1111/dom.13148

319 [19] Nichols GA, Conner C, Brown JB. Initial nonadherence, primary failure and therapeutic 320 success of metformin monotherapy in clinical practice. Curr Med Res Opin 2010;26:2127-35. 321 doi: $10.1185 / 03007995.2010 .504396$

322 [20] Giugliano D, Maiorino MI, Bellastella G, et al. Glycemic control in type 2 diabetes: from 323 medication nonadherence to residual vascular risk. Endocrine 2018;61:23-27. doi:

324 10.1007/s12020-017-1517-9

325 [21] Young LA, Buse JB, Weaver MA, et al. Glucose self-monitoring in non - insulin- treated 326 patients with type 2 diabetes in primary care settings: a randomized trial. JAMA Intern Med 2017. 327 doi:10.1001/jamainternmed.2017.1233

328 [22] International Diabetes Federation. Guideline on self-monitoring of blood glucose in non329 insulin treated type 2 diabetes [Internet]. 2017 Nov 4. Available from: https://www.idf.org/e330 library/guidelines/85-self-monitoring-of-blood-glucose-in-non-insulin-treated-type-2331 diabetes.html. Accessed 2020 Aug 23.

332 [23] Chen GD, Huang CN, Yang YS, et al. Patient perception of understanding health education 333 and instructions has moderating effect on glycemic control. BMC Public Health 2014;14:683. 334 doi: 10.1186/1471-2458-14-683

335 [24] Akter S, Goto A, Mizoue T. Smoking and the risk of type 2 diabetes in Japan: a systematic 336 review and meta-analysis. J Epidemiol 2017;27:553-61.

337 [25]. Ohkuma T, Iwase M, Fujii H, et al. Dose- and time-dependent association of smoking and 338 its cessation with glycemic control and insulin resistance in male patients with type 2 diabetes 339 mellitus: the Fukuoka Diabetes Registry. PLoS One. 2015;10(3):e0122023.

340 doi:10.1371/journal.pone. 0122023 
341 [26] $\square$ liwi $\square$ ska-Mosso $\square$ M, Milnerowicz H. The impact of smoking on the development of

342 diabetes and its complications. Diabetes Vasc Dis Res 2017;14:265-76

343 [27] Yin J, Yeung R, Luk A, et al. Gender, diabetes education, and psychosocial factors are

344 associated with persistent poor glycemic control in patients with type 2 diabetes in the Joint Asia

345 Diabetes Evaluation (JADE) program. J Diabetes 2016;8(1):109-119. doi:10.1111/1753-

$346 \quad 0407.12262$

347 [28] Schütt M, Zimmermann A, Hood R, et al. Gender-specific effects of treatment with lifestyle,

348 metformin or sulfonylurea on glycemic control and body weight: a German multicenter analysis

349 on 9108 patients. Exp Clin Endocrinol Diabetes 2015;123(10):622-626. doi:10.1055/s-0035-

3501559608

351 [29] Casagrande SS. Fradkin JE. Saydah SH. Rust KF , Cowie CC. The prevalence of meeting

352 A1C, blood pressure, and LDL goals among people with diabetes, 1988-2010. Diabetes Care

353 2013;36:2271-2279. doi: 10.2337/dc12-2258

354 [30] McCoy RG, Ngufor C, Van Houten HK, Caffo B, Shah ND. Trajectories of glycemic 355 change in a national cohort of adults with previously controlled type 2 diabetes. Med Care 356 2017;55(11):956-964. doi:10.1097/MLR.0000000000000807

357 [31] Mondesir FL, White K, Liese AD, McLain AC. Gender, illness-related diabetes social 358 support, and glycemic control among middle-aged and older adults. J Gerontol B Psychol Sci 359 Soc Sci 2016;71(6):1081-1088. doi:10.1093/geronb/gbv061

360 [32] Martono DP, Lub R, Lambers Heerspink HJ, et al. Predictors of response in initial users of 361 metformin and sulphonylurea derivatives: a systematic review. Diabet Med 2015;32:853-64. doi:

$36210.1111 /$ dme. 12688

363 [33] Chang AM, Halter JB. Aging and insulin secretion. Am J Physiol Endocrinol Metab 364 2003;284:E7-12. doi: 10.1152/ajpendo.00366.2002

365 [34] Geloneze B, de Oliveira Mda S, Vasques AC, et al. Impaired incretin secretion and 366 pancreatic dysfunction with older age and diabetes. Metabolism 2014;63:922-9. doi:

367 10.1016/j.metabol.2014.04.004

368 [35] Scott G, Ramos-Pérez N, Ramos-Estremera N et al. Relating sociodemographic factors and 369 glycemic control among inpatients with type 2 diabetes. J Public Health (Berl.) 2020.

370 https://doi.org/10.1007/s10389-020-01303-1

371 [36] Benoit SR, Fleming R, Philis-Tsimikas A, et al. Predictors of glycemic control among 
372 patients with Type 2 diabetes: A longitudinal study. BMC Public Health 2005;5:36. doi:

373 10.1186/1471-2458-5-36

374 [37] Wu TY, Majeed A, Kuo KN. An overview of the healthcare system in Taiwan. London J

375 Prim Care (Abingdon) 2010;3(2):115-9. doi: 10.1080/17571472.2010.11493315

376 [38] American Diabetes Association. 9. Pharmacologic approaches to glycemic treatment: standards

377 of medical care in diabetes-2020. Diabetes Care 2020;43(Suppl 1):S98-110. 
Figure 1

Flowchart of the study population.

Abbreviations: $\mathrm{CCH}$, Changhua Christian Hospital; DM, diabetes mellitus; eGFR, estimated glomerular filtration rate. 


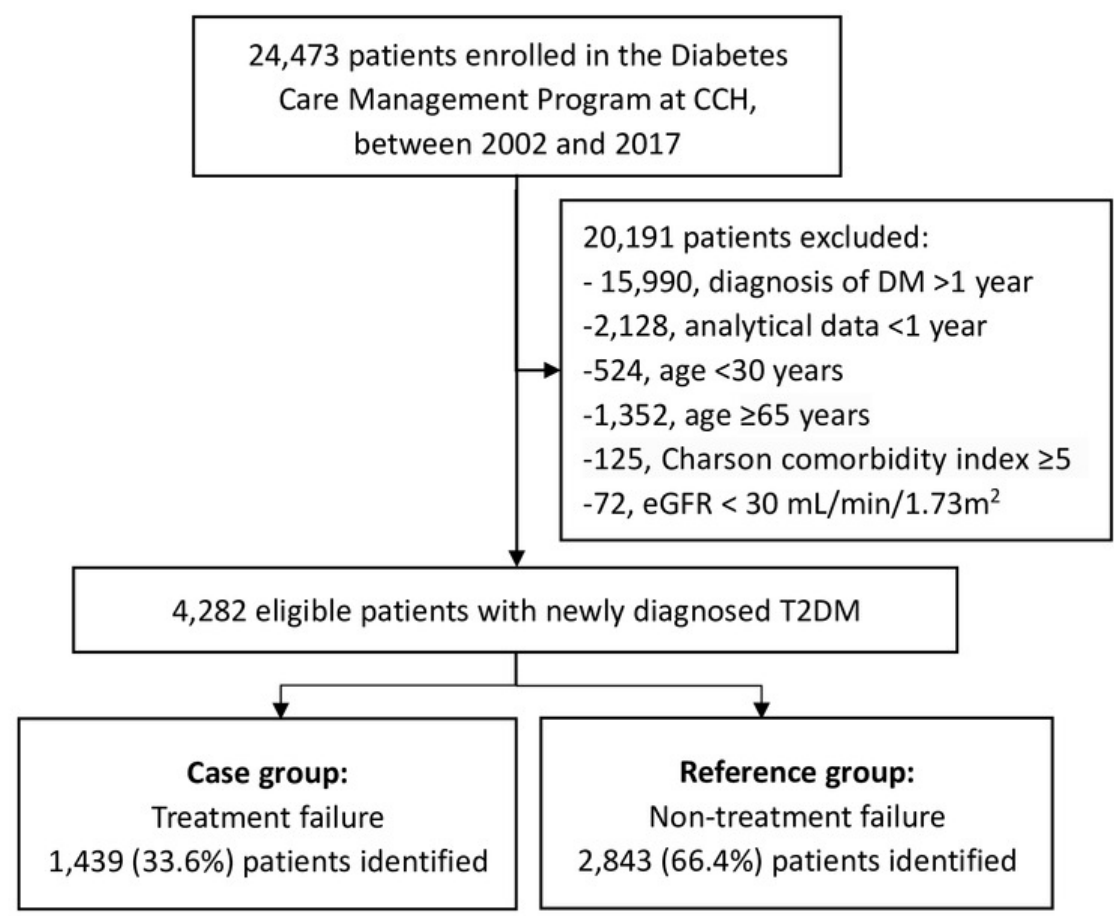




\section{Table $\mathbf{1}$ (on next page)}

Basic characteristics of newly-diagnosed type 2 diabetes patients: TF vs non-TF group.

Notes. Results are expressed as mean \pm SD or $n(\%) .{ }^{a}$ Odds ratio was calculated by per 10 units increase.

Abbreviations: TF, treatment failure; Non-TF, Non-treatment failure; SD, standard deviation; OR, odds ratio; $\mathrm{Cl}$, confidence interval; DM, diabetes mellitus; GC, glycemic control; HbAlc, hemoglobin Alc; BMI, body mass index; BP, blood pressure; HDL-C, high-density lipoprotein cholesterol; LDL-C, low-density lipoprotein cholesterol; eGFR, estimated glomerular filtration rate; GPT, glutamic pyruvic transaminase; OAD, oral antidiabetic drug; $\mathrm{CCl}$, Charlson comorbidity index; CHF, congestive heart failure; CAD, coronary artery disease; CVA, cerebrovascular accident. 


\begin{tabular}{|c|c|c|c|c|}
\hline & $\begin{array}{c}\text { TF } \\
n=1439\end{array}$ & $\begin{array}{c}\text { Non-TF } \\
n=2843\end{array}$ & $\begin{array}{c}\text { OR } \\
(95 \% \mathrm{Cl})\end{array}$ & $p$-value \\
\hline Age at onset (years) & $50.2 \pm 8.5$ & $51.3 \pm 8.5$ & $0.98(0.98,0.99)$ & $<0.001$ \\
\hline Gender: Male & $730(50.7 \%)$ & 1631 (57.4\%) & $0.77(0.67,0.87)$ & $<0.001$ \\
\hline Level of education: No & $112(7.8 \%)$ & $130(4.6 \%)$ & 1 & \\
\hline Primary school & $478(33.2 \%)$ & $803(28.2 \%)$ & $0.69(0.52,0.91)$ & 0.009 \\
\hline High school & $654(45.5 \%)$ & $1282(45.1 \%)$ & $0.59(0.45,0.78)$ & $<0.001$ \\
\hline University or above & $195(13.6 \%)$ & $628(22.1 \%)$ & $0.36(0.27,0.49)$ & $<0.001$ \\
\hline Family history of DM: Yes & 709 (49.3\%) & $1502(52.8 \%)$ & $0.87(0.76,0.98)$ & 0.028 \\
\hline Current smoking & $310(21.5 \%)$ & 492 (17.3\%) & $1.31(1.12,1.54)$ & $<0.001$ \\
\hline Alcohol drinking & $95(6.6 \%)$ & $233(8.2 \%)$ & $0.79(0.62,1.01)$ & 0.064 \\
\hline Physical activity: No exercise & 899 (63.4\%) & 1396 (49.5\%) & 1 & \\
\hline Occasional exercise & $220(15.5 \%)$ & 526 (18.7\%) & $0.65(0.54,0.78)$ & $<0.001$ \\
\hline Regular exercise & $300(21.1 \%)$ & 896 (31.8\%) & $0.52(0.45,0.61)$ & $<0.001$ \\
\hline Knowledge regarding GC: Yes & $710(54.2 \%)$ & $1811(68.7 \%)$ & $0.54(0.47,0.62)$ & $<0.001$ \\
\hline Willingness toward DSM: Yes & 1056 (80.6\%) & $2220(84.3 \%)$ & $0.77(0.65,0.92)$ & 0.004 \\
\hline Perform SMBG: Yes & $270(18.8 \%)$ & 816 (28.7\%) & $0.57(0.49,0.67)$ & $<0.001$ \\
\hline Medication adherence: Yes & $1353(94.0 \%)$ & 2752 (96.8\%) & $0.52(0.38,0.70)$ & $<0.001$ \\
\hline \multicolumn{5}{|l|}{ Clinical variables } \\
\hline HbA1c at baseline (\%) & $9.8 \pm 2.5$ & $8.7 \pm 2.6$ & $1.17(1.15,1.20)$ & $<0.001$ \\
\hline HbA1c at 3-month (\%) & $7.9 \pm 1.6$ & $6.5 \pm 1.0$ & $2.61(2.42,2.81)$ & $<0.001$ \\
\hline HbA1c at 6-month (\%) & $7.9 \pm 1.5$ & $6.3 \pm 0.8$ & $4.91(4.41,5.47)$ & $<0.001$ \\
\hline HbA1c at 9-month (\%) & $8.0 \pm 1.5$ & $6.3 \pm 0.7$ & $8.51(7.42,9.76)$ & $<0.001$ \\
\hline $\mathrm{HbA} 1 \mathrm{c}$ at 12 -month (\%) & $8.3 \pm 1.3$ & $6.2 \pm 0.5$ & -- & -- \\
\hline $\mathrm{BMI}\left(\mathrm{kg} / \mathrm{m}^{2}\right)$ & $26.68 \pm 4.6$ & $26.6 \pm 4.3$ & $1.00(0.99,1.02)$ & 0.569 \\
\hline Mean BP (mmHg) & $97.7 \pm 12.6$ & $96.4 \pm 12.1$ & $1.01(1.00,1.01)$ & 0.001 \\
\hline Total cholesterol (mg/dL) & $192.9 \pm 47.1$ & $181.0 \pm 40.2$ & $1.07(1.05,1.08)^{\mathrm{a}}$ & $<0.001$ \\
\hline Triglycerides (mg/dL) & $182.2 \pm 193.9$ & $152.2 \pm 138.4$ & $1.01(1.01,1.02)^{\mathrm{a}}$ & $<0.001$ \\
\hline $\mathrm{HDL}-\mathrm{C}(\mathrm{mg} / \mathrm{dL})$ & $47.7 \pm 17.2$ & $46.3 \pm 11.9$ & $1.01(1.00,1.01)$ & 0.003 \\
\hline $\mathrm{LDL}-\mathrm{C}(\mathrm{mg} / \mathrm{dL})$ & $113.7 \pm 35.4$ & $106.7 \pm 33.0$ & $1.06(1.04,1.08)^{\mathrm{a}}$ & $<0.001$ \\
\hline eGFR $\left(\mathrm{mL} / \mathrm{min} / 1.73 \mathrm{~m}^{2}\right)$ & $98.5 \pm 41.1$ & $96.5 \pm 30.7$ & $1.02(1.00,1.04)^{\mathrm{a}}$ & 0.076 \\
\hline GPT (U/L) & $36.7 \pm 31.8$ & $34.9 \pm 35.0$ & $1.02(1.00,1.03)^{\mathrm{a}}$ & 0.109 \\
\hline \multicolumn{5}{|l|}{ Anti-diabetic Medication } \\
\hline None or OAD alone & 1203 (83.6\%) & $2550(89.7 \%)$ & 1 & \\
\hline Insulin alone & $63(4.4 \%)$ & $61(2.2 \%)$ & $2.19(1.53,3.13)$ & $<0.001$ \\
\hline OAD+ insulin & $173(12.0 \%)$ & $232(8.2 \%)$ & $1.58(1.28,1.95)$ & $<0.001$ \\
\hline Anti-hypertension agent & $670(46.6 \%)$ & 1345 (47.3\%) & $0.97(0.85,1.10)$ & 0.643 \\
\hline Use of statins & 786 (54.6\%) & 1577 (55.5\%) & $0.97(0.85,1.10)$ & 0.598 \\
\hline Use of fibrates & 215 (14.9\%) & 338 (11.9\%) & $1.30(1.08,1.57)$ & 0.005 \\
\hline
\end{tabular}




\begin{tabular}{lcccc} 
Comorbidity: CCl score & $1.6 \pm 0.9$ & $1.6 \pm 0.9$ & $0.94(0.88,1.02)$ & 0.123 \\
CHF & $119(8.3 \%)$ & $279(9.8 \%)$ & $0.83(0.66,1.04)$ & 0.101 \\
CAD & $82(5.7 \%)$ & $167(5.9 \%)$ & $0.97(0.74,1.27)$ & 0.817 \\
CVA & $57(4.0 \%)$ & $100(3.5 \%)$ & $1.13(0.81,1.58)$ & 0.466 \\
Enrollment time: 2002-2009 & $959(66.6 \%)$ & $1393(49.0 \%)$ & 1 & \\
$2010-2017$ & $480(33.4 \%)$ & $1450(51.0 \%)$ & $0.48(0.42,0.55)$ & $<0.001$ \\
\hline
\end{tabular}

1 


\section{Table 2 (on next page)}

Models to predict treatment failure by multivariable logistic regression analysis using backward elimination method. Primary model: all study participants; Subgroup model: subgroup analysis of patients with baseline $\mathrm{HbAlc} \geq 8 \%$.

Notes. ${ }^{a}$ Odds ratio was calculated by per 10 -unit increase.

Abbreviations: OR, odds ratio; $\mathrm{Cl}$, confidence interval; $\mathrm{HbAlc}$, hemoglobin $\mathrm{Alc}$; $\mathrm{GC}$, glycemic control; SMBG, self-monitoring of blood glucose; BP, blood pressure; OAD, oral anti-diabetic drug; AUC, area under curve. 


\begin{tabular}{|c|c|c|c|c|}
\hline & \multicolumn{2}{|c|}{ Primary model $(n=4282)$} & \multicolumn{2}{|c|}{ Subgroup model $(n=2392)$} \\
\hline & OR $(95 \% \mathrm{Cl})$ & $p$-value & OR $(95 \% \mathrm{Cl})$ & $p$-value \\
\hline HbA1c at baseline (\%) & $1.17(1.14,1.2)$ & $<0.001$ & & \\
\hline Age at onset (years) & $0.98(0.97,0.99)$ & $<0.001$ & & \\
\hline Gender: Men & $0.64(0.54,0.75)$ & $<0.001$ & $0.65(0.54,0.78)$ & $<0.001$ \\
\hline Level of education: No & 1 & & & \\
\hline Primary school & $0.92(0.68,1.26)$ & 0.62 & & \\
\hline High school & $0.77(0.56,1.07)$ & 0.12 & & \\
\hline University or above & $0.55(0.38,0.8)$ & 0.002 & & \\
\hline Current smoking & $1.39(1.14,1.69)$ & 0.001 & & \\
\hline Physical activity: No exercise & 1 & & 1 & \\
\hline Occasional exercise & $0.86(0.71,1.04)$ & 0.13 & $0.91(0.71,1.16)$ & 0.44 \\
\hline Regular exercise & $0.68(0.57,0.81)$ & $<0.001$ & $0.59(0.48,0.74)$ & $<0.001$ \\
\hline Perform SMBG: Yes & $0.73(0.61,0.89)$ & 0.002 & $0.63(0.49,0.8)$ & $<0.001$ \\
\hline Medication adherence: Yes & $0.53(0.36,0.79)$ & 0.002 & $0.38(0.23,0.66)$ & $<0.001$ \\
\hline Mean BP (mmHg) & $1.01(1.00,1.01)$ & 0.014 & & \\
\hline Triglycerides (mg/dL) & $1.01(1.00,1.01)^{a}$ & 0.004 & $1.01(1.00,1.01)^{\mathrm{a}}$ & 0.054 \\
\hline Total cholesterol (mg/dL) & & & $1.03(1.00,1.05)^{a}$ & 0.031 \\
\hline \multicolumn{5}{|l|}{ Anti-diabetic Medication: } \\
\hline None or OAD alone & 1 & & 1 & \\
\hline Insulin alone & $2.15(1.44,3.21)$ & $<0.001$ & $1.97(1.24,3.15)$ & 0.004 \\
\hline OAD+ insulin & $1.35(1.06,1.73)$ & 0.015 & $1.36(1.03,1.79)$ & 0.031 \\
\hline Enrollment time, 2010-2017 & $0.67(0.57,0.79)$ & $<0.001$ & $0.6(0.49,0.73)$ & $<0.001$ \\
\hline R square & 0.136 & & 0.111 & \\
\hline AUC for model & 0.694 & & 0.673 & \\
\hline
\end{tabular}




\section{Table 3 (on next page)}

Correlations between demographic variables and self-care factors for diabetes management.

Notes. Kendall's tau rank correlation coefficient was used. $* p<0.05, * * p<0.01$.

Abbreviations: GC, glycemic control; DSM, diabetes self-management; SMBG, self-monitoring of blood glucose. 


\begin{tabular}{lccccccccc}
\hline Variables & $\mathbf{1}$ & $\mathbf{2}$ & $\mathbf{3}$ & $\mathbf{4}$ & $\mathbf{5}$ & $\mathbf{6}$ & $\mathbf{7}$ & $\mathbf{8}$ & $\mathbf{9}$ \\
\hline 1. Education level & 1 & $0.26^{* *}$ & $0.059^{* *}$ & $0.057^{* *}$ & $0.096^{* *}$ & $0.19^{* *}$ & $-0.44^{* *}$ & $0.26^{* *}$ & $0.055^{* *}$ \\
2. Knowledge regarding GC & 1 & $0.21^{* *}$ & $0.055^{* *}$ & $0.20^{* *}$ & $0.30^{* *}$ & $-0.036^{*}$ & $0.036^{*}$ & $-0.033^{*}$ \\
3. Willingness toward DSM & & & 1 & $0.036^{*}$ & $0.042^{* *}$ & $0.079^{* *}$ & -0.011 & 0.01 & -0.020 \\
4. Medication adherence & & & 1 & $0.053^{* *}$ & $0.043^{* *}$ & -0.008 & 0.02 & -0.006 \\
5. Physical activity & & & & & 1 & $0.16^{* *}$ & $0.14^{* *}$ & 0.019 & $-0.092^{* *}$ \\
6. Perform SMBG & & & & & & 1 & $-0.061^{* *}$ & $0.053^{* *}-0.016$ \\
7. Age (years) & & & & & & & 1 & $-0.11^{* *}$ & $-0.12^{* *}$ \\
8. Gender (Men) & & & & & & & & 1 & $0.39^{* *}$ \\
9. Current smoking & & & & & & & & & 1 \\
\hline
\end{tabular}

